\title{
Magnetoquantum Oscillations at THz Frequencies in InSb
}

\author{
P. Gogoi ${ }^{1}$ D. Kamenskyi, ${ }^{1,2}$ D. D. Arslanov, ${ }^{2}$ R. T. Jongma, ${ }^{2}$ W. J. van der Zande, ${ }^{2}$ B. Redlich, ${ }^{2}$ \\ A. F. G. van der Meer, ${ }^{2}$ H. Engelkamp, ${ }^{1}$ P. C. M. Christianen, ${ }^{1}$ and J. C. Maan ${ }^{1,2}$ \\ ${ }^{1}$ High Field Magnet Laboratory (HFML-EMFL), Radboud University, \\ Toernooiveld 7, 6525 ED Nijmegen, The Netherlands \\ ${ }^{2}$ FELIX Laboratory, Institute for Molecules and Materials, Radboud University, \\ Toernooiveld 7c, 6525 ED Nijmegen, The Netherlands
}

(Received 23 December 2016; published 6 October 2017)

\begin{abstract}
The ac magnetoconductance of bulk InSb at $\mathrm{THz}$ frequencies in high magnetic fields, as measured by the transmission of $\mathrm{THz}$ radiation, shows a field-induced transmission, which at high temperatures $(\approx 100 \mathrm{~K})$ is well explained with classical magnetoplasma effects (helicon waves). However, at low temperatures (4 K), the transmitted radiation intensity shows magnetoquantum oscillations that represent the Shubnikov-de Haas effect at THz frequencies. At frequencies above $0.9 \mathrm{THz}$, when the radiation period is shorter than the Drude scattering time, an anomalously high transmission is observed in the magnetic quantum limit that can be interpreted as carrier localization at high frequencies.
\end{abstract}

DOI: 10.1103/PhysRevLett.119.146603

Modern electronic devices, such as Si-based processors and memories in computers, work at several $\mathrm{GHz}$, while $\mathrm{THz}$ electronics is contemplated for devices made from graphene [1]. From a fundamental point of view, $\mathrm{THz}$ experiments represent a new electronic ac conductivity regime [2] since the typical electron scattering rates become comparable to the excitation frequencies. $\mathrm{THz}$ frequencies correspond to photon energies of a few $\mathrm{meV}$, and conductivity is measured as the (far infrared) optical response rather than the standard electrical ac conductivity. To study this new transport regime, we performed $\mathrm{THz}$ magnetotransmission experiments on heavily doped InSb (a narrowgap, light mass semiconductor with high electron mobility $[3,4])$ in very high magnetic fields.

Optical properties of materials are determined by the dielectric tensor $\epsilon$ (proportional to the square of the complex refractive index $\tilde{n}$ ) $[5,6]$ :

$$
\tilde{n}^{2}=\epsilon(\omega, B)=\kappa+\frac{i \tilde{\sigma}(\omega, B)}{\omega \epsilon_{0}}+\epsilon_{\mathrm{ph}}
$$

$\tilde{\sigma}(\omega, B)$ is the complex conductivity tensor implying that ac conductivity can be measured by optical means. $B$ is the magnetic field strength, $\omega$ is the angular frequency, and $\epsilon_{0}$ is the permittivity of free space. The lattice contribution to the dielectric tensor consists of two terms. $\kappa$ is the dielectric permittivity $\left(\kappa=16\right.$ for InSb [3]), and $\epsilon_{\mathrm{ph}}$ describes the resonant contribution of the optical phonons that are present in polar materials, such as InSb, interacting with the plasmon [5]. We include $\epsilon_{\mathrm{ph}}$ in the harmonic oscillator approximation: $\epsilon_{\mathrm{ph}}=\left(\omega_{L}^{2}-\omega_{T}^{2}\right) \kappa /\left(\omega_{T}^{2}-\omega^{2}-i \nu_{\mathrm{ph}} \omega\right)$. Here, $\omega_{L}$ and $\omega_{T}$ are the angular frequencies of the longitudinal and transverse optical phonons, which are 196.8 and $184.8 \mathrm{~cm}^{-1}$, respectively, for InSb. $\nu_{\mathrm{ph}}$ is the phonon scattering rate $\left(33.3 \mathrm{~cm}^{-1}\right)$ [7]. In the Faraday configuration (when the magnetic field is applied along the wave propagation) Eq. (1) can be written for circularly polarized light as [5]

$n_{ \pm}^{2}=\epsilon_{ \pm}=\kappa+\frac{i}{\omega \epsilon_{0}}\left[\sigma_{x x}(\omega, B) \mp i \sigma_{x y}(\omega, B)\right]+\epsilon_{\mathrm{ph}}$.

$\sigma_{x x}(\omega, B)$ and $\sigma_{x y}(\omega, B)$ are, respectively, the real longitudinal and transversal components of the conductivity. The + and - signs denote left- $(+)$ and right-handed $(-)$ circularly polarized light.

In our experiments, $\mathrm{THz}$ radiation was generated by a free electron laser (FEL) covering the frequency range $6-100 \mathrm{~cm}^{-1}(0.2-3.0 \mathrm{THz})$ and radiation powers up to $200 \mathrm{~W} \mathrm{~cm}^{-2}$ [8-11]. The sample transmission was measured in a magnetic field (up to $33 \mathrm{~T}$ ) in the Faraday geometry as shown in Fig. 1(a) [12]. The sample was a $0.63 \times 10^{2} \mathrm{~mm}^{3}$ large crystal of Te-doped $\left(\begin{array}{lll}1 & 0 & 0\end{array}\right) n$-InSb with electron density $N=1.66 \times 10^{17} \mathrm{~cm}^{-3}$ and mobility $\mu=8 \times 10^{4} \mathrm{~cm}^{2} \mathrm{~V}^{-1} \mathrm{~s}^{-1}$ (at $4.2 \mathrm{~K}$ ), as obtained from Hall effect measurements, corresponding to a scattering rate $\nu=1.05 \mathrm{THz}$. For this electron density, the Fermi energy $E_{F}=50 \mathrm{meV}$ lies inside the conduction band. The electron effective mass $m^{*}=0.021 m_{0}\left(m_{0}\right.$ is the free electron mass) and the effective $g$ factor $g=33.5 \pm 1.0$ at $E_{F}$ are obtained from the temperature dependence of the Shubnikov-de Haas (SdH) quantum oscillations. These values are in good agreement with literature values [15-17], including the effect of conduction band nonparabolicity as described by the Kane model $[18,19]$.

The plasma frequency equals $\omega_{p}=\sqrt{e^{2} N / m^{*} \kappa \epsilon_{0}} \approx$ $40 \mathrm{THz}\left(211 \mathrm{~cm}^{-1}\right)$, which is higher than our measuring frequencies $\left(6-100 \mathrm{~cm}^{-1}\right)$, making the sample nontransparent at zero field [solid lines in Figs. 1(b) and 1(c)]. With 

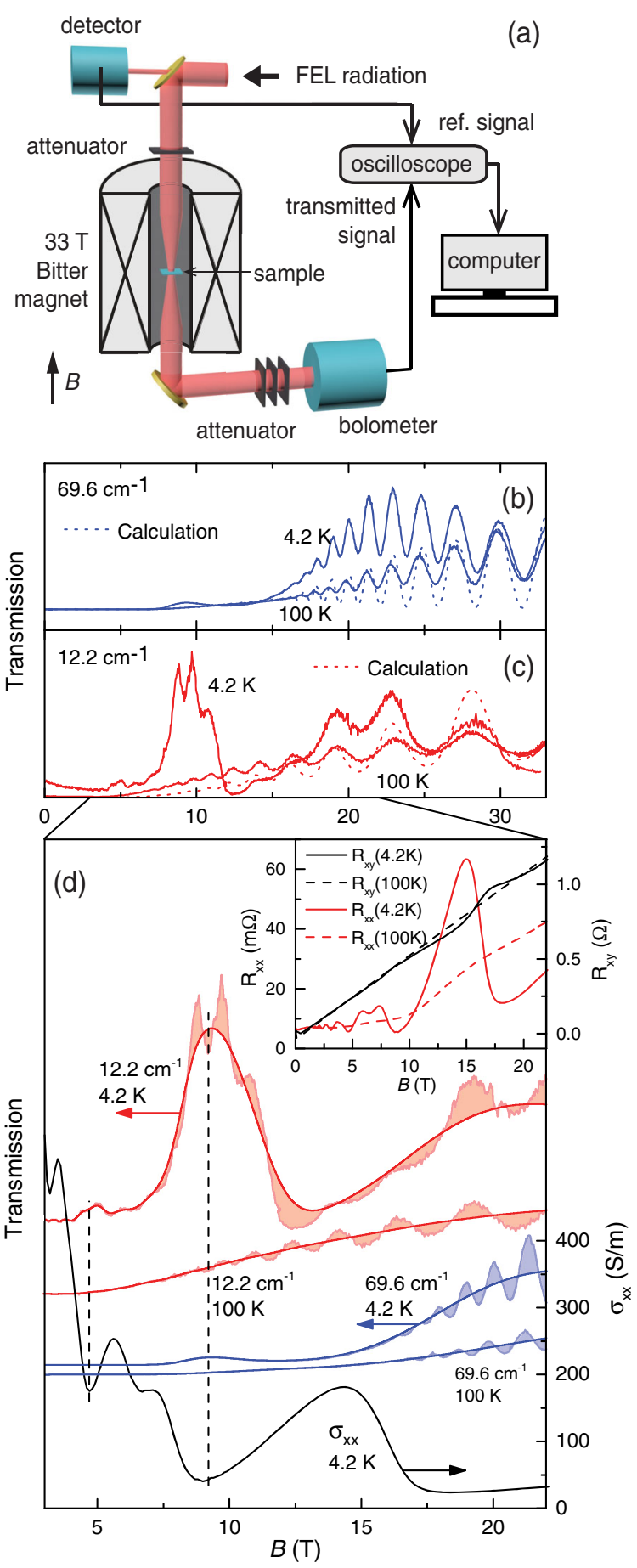

FIG. 1. (a) Schematic experimental setup. (b),(c) Measured transmission signal of InSb as a function of the magnetic field at (b) $12.2 \mathrm{~cm}^{-1}$ and (c) $69.6 \mathrm{~cm}^{-1}$ at 100 and $4.2 \mathrm{~K}$. The dotted curves correspond to theoretical calculations [using Eq. (3)]. (d) The same data as in (b),(c) but with the rapid Fabry-Pérot oscillations filtered out (solid lines; the filled areas show the original data [12]). The black solid curve at the bottom of (d) shows the longitudinal conductivity as a function of magnetic field at $4.2 \mathrm{~K}$. Minima in $\sigma_{x x}$ (vertical dashed lines) coincide with maxima in transmission at low temperatures. Inset: Measured longitudinal (red) and Hall (black) dc resistances at $4.2 \mathrm{~K}$ (solid lines) and $100 \mathrm{~K}$ (dashed lines). increasing magnetic field, transparency sets in and shows a rapidly varying oscillatory structure that is temperature independent but which has a period that is frequency dependent. At high temperatures, the average transmission increases smoothly with increasing magnetic field [Figs. 1(b) and 1(c)], while at low temperatures, the transmission amplitude is modulated in phase with the magnetoquantum oscillations of the dc conductivity [Fig. 1(d)].

To analyze the high temperature data, we rewrite Eq. (2) by using the classical Drude conductivity $[5,6]$ :

$$
n_{ \pm}^{2}=\epsilon_{ \pm}=\kappa\left(1-\frac{\omega_{p}^{2}}{\omega\left(\omega \pm \omega_{c}+i \nu\right)}\right)+\epsilon_{\mathrm{ph}},
$$

with $\omega_{c}=e B / m^{*}$ the cyclotron frequency. Using this equation, we have calculated the high temperature transmittance for unpolarized (i.e., equal intensities of left and right circular polarizations) incident light [12]. We used the known sample parameters and a fixed scattering rate as obtained from the dc conductivity. A small and known frequency spread $\left(\sim 0.5 \mathrm{~cm}^{-1}\right)$ from the FEL is included in $\omega$. The results [dotted lines in Figs. 1(b) and 1(c)] explain the $100 \mathrm{~K}$ data very well. Two main contributions to the signal can be distinguished [12]. Focusing on the first (plasma) term in Eq. (3) and neglecting the scattering rate $\nu$ (the lossless case) shows that $\epsilon_{ \pm}$is negative (i.e., imaginary refractive index) at zero magnetic field, resulting in an opaque sample for frequencies below the plasma frequency. At a certain field strength, the cyclotron frequency $\omega_{c}$ becomes higher than the measuring frequency $\omega$, and for the negative (-) sign in Eq. (3) [the cyclotron resonance active (CRA) mode], $\epsilon_{-}$becomes positive, making the sample transparent (an effect known as helicon wave propagation $[20,21])$. The data for the lowest frequency $\left[12.2 \mathrm{~cm}^{-1}\right.$, Fig. 1(b)] are in this regime when $\omega_{c}>\omega$ for $B>0.27 \mathrm{~T}$. The rapid almost temperature-independent oscillations at higher fields are due to Fabry-Pérot interferometerlike resonances caused by the strongly field-dependent refractive index. The interference occurs when an integer number of half wavelengths $\left(\lambda_{\text {sample }}=\right.$ $\lambda_{\text {ext }} / n_{ \pm}$) equals the sample thickness $d$. Scanning the field, successive maxima and minima occur, as indeed observed [Fig. 1(b)]. These helicon waves are the first contribution to the transmission signal. The second contribution becomes visible at higher frequencies, such as $69.6 \mathrm{~cm}^{-1}$ in Fig. 1(c), where the denominator in Eq. (3) becomes larger than $\omega_{p}$, making $n_{+}$[the cyclotron resonance inactive (CRI) mode] also real, and both CRI and CRA polarization modes are transmitted. In this situation, an additional Fabry-Pérot-type effect occurs, arising from interference between the CRI and CRA polarizations for a given thickness that produces Faraday rotations [5,12]. The field-induced oscillations observed at $69.6 \mathrm{~cm}^{-1}$ in Fig. 1(c) are mainly caused by this Faraday rotation effect [12]. 
Whereas the high temperature data, as shown above, can be explained properly by Eq. (3), at low temperatures $(4.2 \mathrm{~K})$, the measured amplitudes deviate significantly from the calculations. In particular, the slowly varying envelope of the broad transmission with peaks around 5, 9, and $21 \mathrm{~T}$ [Fig. 1(d)] is not reproduced. For the electron density in our sample, above $18 \mathrm{~T}$, only the lowest $(N=0)$ Landau level (LL) with spin-up electrons is occupied (extreme quantum limit). With decreasing field, the minima correspond to the population of, successively, the spin-down electrons in the lowest $(N=0) \mathrm{LL}(9.2 \mathrm{~T})$, the spin-up states of the $N=1$ LL (shallow minimum at $7 \mathrm{~T}$ ), and the spin-down electrons of the $N=1 \mathrm{LL}(4.8 \mathrm{~T})$. This changing occupancy of the LLs gives rise to the well-known SdH oscillations in the resistivity [inset of Fig. 1(d)] and the corresponding conductivity [bottom black solid line in Fig. 1(d)]. It can clearly be seen that low conductance values correspond to a high transmission through the sample (dotted vertical lines); i.e., the envelope of the field-dependent transmission has a slowly varying field dependence that mimics the $\mathrm{SdH}$ oscillation in the dc conductivity. Since we observe magnetoquantum oscillations in the $\mathrm{THz}$ transmission, the simple classical Drude conductivity used in Eq. (3) is inadequate to describe the low temperature data.

Figure 2 shows the normalized transmission data (i.e., normalized to the empty sample holder transmission) at $4.2 \mathrm{~K}$ for a wide range of frequencies. As before, the fieldinduced transparency is observed with the rapid oscillations, having a faster periodicity with increasing $\mathrm{THz}$ frequencies. Extra transmission peaks are observed around $5 \mathrm{~T}$, at low frequencies, and at $9 \mathrm{~T}$ for all $\mathrm{THz}$ frequencies [see the inset of Fig. 2(a)]. The peaks in transmission occur at field values corresponding to conductance minima [as in Fig. 1(d)]

To analyze these low temperature data in more detail, we return to the general expression of the complex refractive index, as given in Eq. (2). We incorporate, instead of the Drude conductivity, the measured field-dependent dc values for $\sigma_{x x}(0, B)$ and $\sigma_{x y}(0, B)$. In this framework, the real and imaginary parts of $\epsilon_{ \pm}$are given by

$$
\begin{aligned}
& \operatorname{Re}\left(\epsilon_{ \pm}\right) \approx \kappa \pm \frac{1}{\omega \epsilon_{0}} \sigma_{x y}(0, B)+\operatorname{Re}\left(\epsilon_{\mathrm{ph}}\right), \\
& \operatorname{Im}\left(\epsilon_{ \pm}\right) \approx \frac{1}{\omega \epsilon_{0}} \sigma_{x x}(0, B)+\operatorname{Im}\left(\epsilon_{\mathrm{ph}}\right) .
\end{aligned}
$$

$\sigma_{x y}(0, B)$ describes the dispersion and, thus, the period of the Fabry-Pérot maxima. $\sigma_{x y}(0, B)$ is hardly temperature dependent, and indeed, no change in the position of the rapidly varying peaks is observed. The amplitude is determined by the longitudinal conductivity $\sigma_{x x}(0, B)$, which develops magnetoquantum oscillations at low temperatures, and these appear as a slow modulation of the transmission in phase with the $\mathrm{SdH}$ oscillations.

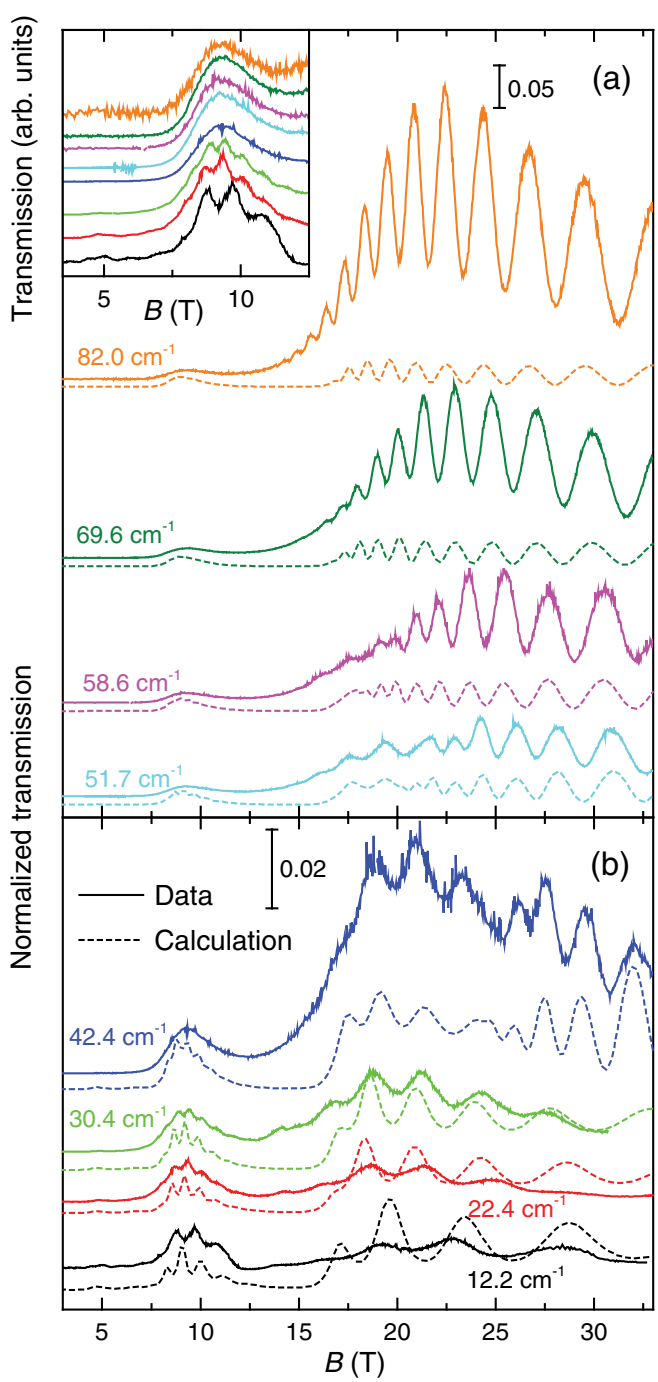

FIG. 2. Normalized transmission of $\mathrm{InSb}$ as a function of magnetic field (solid lines) at $4.2 \mathrm{~K}$ for the FEL frequencies indicated. Scale bars for (a) and (b) are, respectively, $0.05 \%$ and $0.02 \%$. The dotted lines correspond to the calculated transmission [using Eq. (4)] normalized to the peak in the experimental curve around $9 \mathrm{~T}$. In the inset of (a), an enlargement of the low field data is shown, where the same coloring is used as in the (a) and (b) panels.

The dotted lines in Fig. 2 show results of this model (for more details, see the Supplemental Material [12]). The rapidly varying transmission oscillations due to the FabryPérot effect are very well reproduced over the entire field range. The calculated curves properly include the combined effect of the field-dependent refractive index and the Faraday rotation resonances. For instance, for $42.4 \mathrm{~cm}^{-1}$ around $26 \mathrm{~T}$ [blue curve in Fig. 2(b)], a clear change in the oscillation period can be seen, both experimentally and theoretically, which is a direct result of the competition between the two effects [12]. Most important, our model reproduces the magnetoquantum $\mathrm{SdH}$ oscillations, corresponding to the conductance minima around 5,9 , and $18 \mathrm{~T}$ 
in the THz ac conductivity. We would like to stress that our results directly show magnetoquantum oscillations in the real part of the bulk $\mathrm{THz}$ plasma conductivity. In older literature, the observation of ac SdH-type oscillations has been considered theoretically and been hinted at experimentally [21-24]. However, the range of fields and frequencies presented here and the quantitative agreement with our model have not been reported before. Moreover, our results differ from the optical observation of the $\mathrm{SdH}$ effect caused by nonresonant absorption or modulation of the reflectivity at low frequencies, far from the magnetic quantum limit, due to the oscillations in the dc resistance [25]. This remarkable result is particularly surprising because our model relies on a very strong and rather speculative approximation. It implies two important assumptions: namely, (i) the Shubnikov-de Haas effect, as a function of $B$, both for dc as well as for ac conductivity, can be described by a field-dependent dc conductivity, and (ii) this dependence is the same for all frequencies. We are well aware that $\mathrm{SdH}$ oscillations are not simply related to a field-dependent dc mobility but rather are caused by the different scattering angles at different magnetic fields and, furthermore, that CRI and CRA ac excitation modes correspond effectively to a rotating alternating electric field interacting with the carriers. One aspect of the $\mathrm{SdH}$ effect, however, is included in our analysis: the density of states at the Fermi energy oscillates as a function of magnetic field strength, leading to oscillations in both the $\mathrm{dc}$ and ac conductance. However, a more thorough description would require a more sophisticated theory for ac transport.

As seen from Fig. 2, at lower frequencies 30.4, 22.4, and $12.2 \mathrm{~cm}^{-1}$, the agreement between the calculated and measured transmission is very good, justifying our assumption that the ac and dc scattering times are indeed equal. However, at the highest frequencies, deviations occur in the amplitudes. To clarify this behavior, Fig. 3 shows the transmission peak values at $9 \mathrm{~T}\left(I_{1}\right)$ and $19 \mathrm{~T}$ $\left(I_{2}\right)$, where we took the peak value of the smoothed average transmission (as indicated by the arrows in the inset). The calculated amplitude at $9 \mathrm{~T}\left(I_{1}\right)$ is about $4 \%$ at $12.2 \mathrm{~cm}^{-1}$ and decreases by about a factor of 2 at higher frequencies (filled circles in Fig. 3). Experimentally, an amplitude of about $1 \%$ is observed, which is more or less constant for all frequencies (open circles). Even though the agreement between experiment and calculation is not perfect, the overall trend is the same.

In contrast, the calculated $19 \mathrm{~T}$ transmission $\left(I_{2}\right)$ is about $6 \%$ at low frequency and decreases to $3 \%$ at high frequencies, while experimentally, a strong increase from a few percent to $25 \%$ is observed (above $30 \mathrm{~cm}^{-1}$ ) (triangles in Fig. 3). This deviation is related to the ac damping since the Fabry-Pérot oscillations keep the same periodicity. Our data suggest, therefore, a strong frequency dependence of the high frequency magnetoresistance. As is clear from Fig. 3, this deviation occurs for frequencies higher than

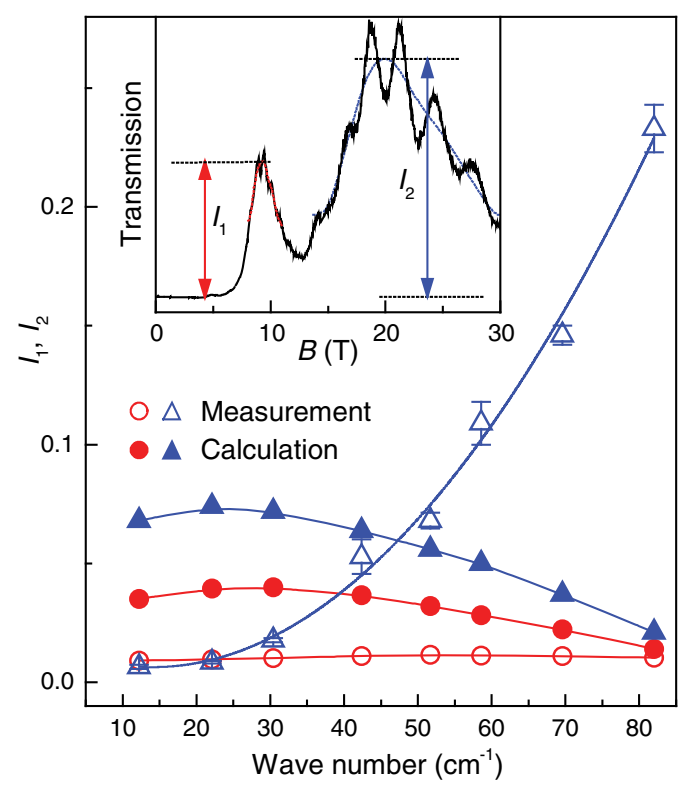

FIG. 3. Measured (open symbols) and calculated (filled symbols) transmission amplitudes at $9 \mathrm{~T}$ (red circles, $I_{1}$ ) and $19 \mathrm{~T}$ (blue triangles, $I_{2}$ ) at $4.2 \mathrm{~K}$. The red and blue solid lines are drawn to guide the eye. The inset shows how the average amplitudes $\left(I_{1}, I_{2}\right.$; dashed curves) are determined from the experimental data (solid curves).

$30 \mathrm{~cm}^{-1}$, which corresponds to $0.9 \mathrm{THz}$ or to a period of $1.1 \times 10^{-12} \mathrm{~s}$, which is actually identical to the transport scattering time calculated from the measured dc electron mobility. Therefore, above $30 \mathrm{~cm}^{-1}$, the scattering time in the magnetic quantum limit becomes longer than the period of the $\mathrm{THz}$ frequencies; i.e., the ac electric field reverses before carriers can scatter, and this effectively suppresses the transport. This ac effective localization in the quantum limit is believed to give rise to an increased transparency, since the carriers cannot effectively couple to the light. This ac-induced localization is very different from known localization effects in the quantum limit. When the cyclotron energy exceeds the thermal energy $\left(\hbar \omega_{c} \gg k T\right)$ and when only the lowest Landau level is occupied, dc (i.e., $\omega=0$ ) magnetotransport may show carrier freeze-out [26] or interaction-induced localization [27]. These effects would show up as a sharp increase in the longitudinal and transverse resistivity, which we do not observe due to the relatively high carrier density in our sample.

In conclusion, we have shown that the transmission of far infrared $(\mathrm{THz})$ radiation in $\mathrm{InSb}$ in high magnetic fields and at low temperatures shows magnetoquantum oscillations demonstrating the $\mathrm{SdH}$ effect at $\mathrm{THz}$ frequencies. In addition, at high magnetic fields and at frequencies $>0.9 \mathrm{THz}$, the observed transmission increases much more than expected, suggesting that the high field and high frequency magnetoresistance shows a transition to an insulating state when the measuring frequency approaches the scattering rate. These studies of magnetoplasma effects 
in $\mathrm{InSb}$ at $\mathrm{THz}$ frequencies and at high magnetic fields provide new information on the $\mathrm{THz}$ ac transport, which we hope inspires theoretical studies.

We acknowledge the support from FELIX, HFML-RU/ FOM, and the Stichting Fundamenteel Onderzoek der Materie with financial support from the Nederlandse Organisatie voor Wetenschappelijk Onderzoek. HFML is a member of the European Magnetic Field Laboratory. FLARE is part of the NCAS project funded through the Big Facilities program of the NWO.

[1] B. Sensale-Rodriguez, R. Yan, M. M. Kelly, T. Fang, K. Tahy, W. S. Hwang, D. Jena, L. Liu, and H. G. Xing, Nat. Commun. 3, 780 (2012).

[2] Z. Mics, K.-J. Tielrooij, K. Parvez, S. A. Jensen, I. Ivanov, X. Feng, K. Müllen, M. Bonn, and D. Turchinovich, Nat. Commun. 6, 7655 (2015).

[3] X. Wang, A. A. Belyanin, S. A. Crooker, D. M. Mittleman, and J. Kono, Nat. Phys. 6, 126 (2010).

[4] T. Arikawa, X. Wang, A. A. Belyanin, and J. Kono, Opt. Express 20, 19484 (2012).

[5] E. Palik and J. Furdyna, Rep. Prog. Phys. 33, 1193 (1970).

[6] P. Y. Liu and J. C. Maan, Phys. Rev. B 47, 16279 (1993).

[7] O. Madelung, Semiconductors: Data Handbook, 3rd ed. (Springer-Verlag, New York, 2004).

[8] R. T. Jongma, A. J. A. Van Roij, R. W. Lof, F. J. P. Wijnen, V.Zhaunerchyk, A. C. N. Engels, G. F. A. J. Wulterkens, P. A. W. van Dael, A. P. van Vliet, W. J. van der Zande, A. F. G. van der Meer, U. Lehnert, R. Wünsch, W. Seidel, P. Michel, K. Dunkel, and C. Piel, in Proceedings of FEL2009, (Liverpool, UK, 2009), p. 435.

[9] V. Zhaunerchyk, R. T. Jongma, Y. Lurie, Y. Pinhasi, and W. J. van der Zande, Appl. Phys. Lett. 97, 231109 (2010).

[10] V. Zhaunerchyk, D. Oepts, R. T. Jongma, and W. J. van der Zande, Phys. Rev. ST Accel. Beams 15, 050701 (2012).
[11] M. Ozerov, B. Bernáth, D. Kamenskyi, B. Redlich, A. F. G. v. d. Meer, P. C. M. Christianen, H. Engelkamp, and J. C. Maan, Appl. Phys. Lett. 110, 094106 (2017).

[12] See Supplemental Material at http://link.aps.org/ supplemental/10.1103/PhysRevLett.119.146603 for details on experimental methods, data filtering, and model calculations, which includes Refs. [13,14].

[13] A. Liptuga, V. Morozhenko, and V. Pipa, Transmission, reflection and thermal radiation of a magneto-optical fabryperot resonator in magnetic field: Investigations and applications, in Infrared Radiation, edited by V. Morozhenko (InTech, Croatia, 2012).

[14] B. Donovan and T. Medcalf, Br. J. Appl. Phys. 15, 1139 (1964).

[15] E. N. Adams and T. D. Holstein, J. Phys. Chem. Solids 10, 254 (1959).

[16] P. Byszewski, J. Koodziejczak, and S. Zukotyski, Phys. Status Solidi (b) 3, 1880 (1963).

[17] A. E. Stephens, D. G. Seiler, J. R. Sybert, and H. J. Mackey, Phys. Rev. B 11, 4999 (1975).

[18] E. O. Kane, J. Phys. Chem. Solids 1, 249 (1957).

[19] B. Lax, J. G. Mavroides, H. J. Zeiger, and R. J. Keyes, Phys. Rev. 122, 31 (1961).

[20] B. W. Maxfield, Am. J. Phys. 37, 241 (1969).

[21] J. K. Furdyna, Appl. Opt. 6, 675 (1967).

[22] B. Aronzon and E. Meilikhov, Zh. Eksp. Teor. Fiz. 67, 277 (1975) [Sov. Phys. JETP 40, 139 (1975)].

[23] B. A. Aronzon and E. Z. Meilikhov, Phys. Status Solidi (b) 79, 753 (1977).

[24] J. K. Furdyna and A. R. Krauss, Phys. Rev. B 2, 3183 (1970).

[25] M. von Ortenberg, Physica (Amsterdam) 177B, 446 (1992), and references cited therein.

[26] M. Shayegan, V. J. Goldman, and H. D. Drew, Phys. Rev. B 38, 5585 (1988).

[27] S. D. Jog and P. R. Wallace, J. Phys. C 11, 2763 (1978). 